\author{
Military Technical College \\ Kobry El-Kobba \\ Cairo, Egypt
}

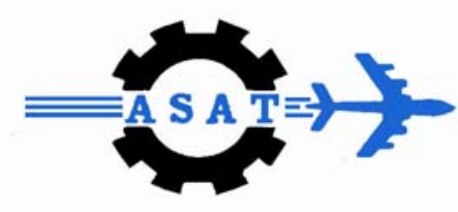

12-th International Conference

on

Aerospace Sciences \&

Aviation Technology

\title{
A RECURRENCE TECHNIQUE FOR COMPUTING THE EFFECTIVE INDEXES OF THE GUIDED MODES OF COUPLED SINGLE-MODE WAVEGUIDES
}

\author{
Tarek A. Ramadan
}

\begin{abstract}
The recurrence dispersion equation of coupled single-mode waveguides is modified by eliminating redundant singularities from the dispersion function. A recurrence zerobracketing (RZB) technique is proposed in which the zeros of the dispersion function at one recurrence step bracket those of the next recurrence step. Numerical examples verify the utility of the RZB technique in computing the roots of the dispersion equation of the TE and TM modes of both uniform and non-uniform arrays.
\end{abstract}

\section{INTRODUCTION}

Single-mode waveguide arrays are widely used in many photonic devices, including directional couplers, modulators, switches, arrayed waveguide gratings, modal and power splitters. In most of these applications the device functionality depends primarily on the interaction of guided, as opposed to leaky or radiation, modes [1].

Many methods have been used to determine the modal properties of waveguide arrays by solving for the roots of the dispersion function, e.g. in [2]-[6]. All of these methods require initial guess for each root without specifying a rule to identify this guess, except for the argument principle method [5], [6]. This method uses the roots of a polynomial as initial guess and then continues to use traditional zero-search techniques [7] to get the actual roots of the dispersion equation. However, the computer implementation of this method is not easy as it involves numerical integration along closed contour in the complex plane. In spite of the applicability of most of the above-mentioned methods to general multilayer structures, it remains desirable to trade this generality in favor of simplicity for more widely used structures such as coupled single-mode waveguides. This simplicity implies developing an efficient zero-search technique, which enables locating the roots of the dispersion equation without using extensive and/or complex numerical computations. In this paper, the recurrence dispersion equation of coupled single-mode waveguides is modified to remove redundant singularities from the dispersion function. A recursive zero-bracketing (RZB) technique is proposed for the computation of the roots of the modified equation.

* Department of Physics, Faculty of Science, Kuwait University, P.O. Box 5969, Safat-13060, Kuwait

(e-mail: tar10@kuc01.kuniv.edu.kw) 


\section{RECURRENCE DISPERSION RELATION}

Different recurrence approaches have been used to express the dispersion relation of waveguide arrays, e.g. in [4] and [8]. The main advantage of these approaches is that they are simple to implement on computers and require fewer computational steps compared to other more complex approaches. Also, they enable monitoring the evolution of modal spectrum with each recurrence step, which not only gives an insight to the physics of modal formation in the array but also is the basis of the proposed RZB technique. In this paper we follow the approach in [4] which applies for both uniform and non-uniform arrays. According to this approach the dispersion relation of an array of $M$ coupled waveguides (see Fig.1) is given by,

$$
\varepsilon_{M}=0
$$

where, $\varepsilon_{M}$, is an implicit dispersion function of the modal effective index, $N$, and the free-space propagation constants, $k_{o}$. It satisfies the recurrence relation,

$$
\varepsilon_{i+1}=J_{i+1} \varepsilon_{i}-\mathrm{K}_{i+1} \varepsilon_{i-1},
$$

where, $i$ is a recurrence index which is incremented in steps from $i=1$ to $i=M-1$.

Even under single-mode condition of the isolated waveguides in the array, the dispersion function, $\varepsilon_{M}$, has singularities in the effective index, $N$. These singularities set up a fundamental zero-bracketing problem [7]. For example, the opposite signs of the dispersion function between two successive values of $N$ may bracket a pole, instead of a zero, due to the discontinuity of that function. In this case, the zero search algorithm may end up returning incorrect roots of the dispersion equation. An example of such an algorithm is that of the FZERO built-in function in MATLAB, which is a widely used software package [9].

As a preliminary step to remove singularities from $\varepsilon_{M}$, we use normalization. The standard normalization parameters used are, $b=\left(N^{2}-n_{s}^{2}\right) /\left(n_{f}^{2}-n_{s}^{2}\right), V_{i}=k_{o} d_{i} \sqrt{n_{f}^{2}-n_{s}^{2}}$, and $V_{i, i+1}=k_{o} d_{i, i+1} \sqrt{n_{f}^{2}-n_{s}^{2}}$. As will be clear shortly, $n_{f}$ is chosen to be the minimum core refractive index in the array, while $n_{s}$ is the substrate refractive index, $d_{i}$ is the width of the $i^{\text {th }}$ waveguide, and $d_{i, i+1}$ is the separation between the $i^{\text {th }}$ the $(i+1)^{\text {th }}$ waveguides. In terms of these normalized parameters the recurrence parameters, $J_{i+1}$ and $\mathrm{K}_{i+1}$, are given by,

$$
\begin{gathered}
J_{i+1}=\frac{\left(\cot \left(\Phi_{i+1}\right)-p_{i+1}\right)+\mu_{i, i+1}\left(\cot \left(\Phi_{i}\right)+p_{i}\right) e^{-2 V_{i, i+1} \sqrt{b}}}{\left(1+p_{i+1} \cot \left(\Phi_{i+1}\right)\right)} \\
\mathrm{K}_{i+1}=\mu_{i, i+1} \frac{\csc ^{2}\left(\Phi_{i}\right)}{\left(1+p_{i} \cot \left(\Phi_{i}\right)\right)\left(1+p_{i+1} \cot \left(\Phi_{i+1}\right)\right)} e^{-2 V_{i, i+1} \sqrt{b}},
\end{gathered}
$$

with

$$
p_{i}=\frac{a_{i}-\left(1+\eta_{i}^{2}\right) b}{2 \eta_{i} \sqrt{b\left(a_{i}-b\right)}},
$$




$$
\mu_{i, i+1}=\frac{\eta_{i}}{\eta_{i+1}} \sqrt{\frac{a_{i}-b}{a_{i+1}-b}} \frac{a_{i+1}+\left(\eta_{i+1}^{2}-1\right) b}{a_{i}+\left(\eta_{i}^{2}-1\right) b},
$$

where $\quad \varepsilon_{0}=1, \quad \varepsilon_{1}=\left(\cot \left(\Phi_{1}\right)-p_{1}\right) /\left(1+p_{1} \cot \left(\Phi_{1}\right)\right), \quad \Phi_{i}=V_{i} \sqrt{a_{i}-b}, \quad$ and $a_{i}=\left(n_{i}^{2}-n_{s}^{2}\right) /\left(n_{f}^{2}-n_{s}^{2}\right)$. Here, $n_{i}$ is the core refractive index of the $i^{\text {th }}$ waveguide, and $\eta_{i}$, depends on the modal polarization. It equals $\left(n_{i} / n_{s}\right)^{2}$ for TM modes and unity for TE modes. By combining (1c)-(1f) with (1b), $\varepsilon_{M}$ becomes a function of $b$ and the parameters, $V_{i}, V_{i, i+1}, a_{i}$, and $\eta_{i}$. In order to identify the singularities of $\varepsilon_{M}$ it is required to identify the possible values of these parameters. Under single-mode condition of the isolated waveguides in the array, $V_{i} \sqrt{a_{i}}<\pi$. The mode-polarization parameter, $\eta_{i} \geq 1$, and the normalized separation, $0<V_{i, i+1}<\infty$. The choice of $n_{f}=\min _{i} n_{i}$ ensures that $a_{i} \geq 1$ for all the waveguides in the array. Also, it implies limiting $b$ between 0 and 1 in most practical applications, where $n_{s} \leq N \leq \min _{i} n_{i}$.

The above constraints on $\eta_{i}, a_{i}$ and $b$ are sufficient to eliminate any poles in $p_{i}$ and $\mu_{i, i+1}$; see (1e) and (1f). Also the single-mode condition, $V_{i} \sqrt{a_{i}}<\pi$, ensures that $\cot \left(\Phi_{i}\right)$ has no poles in the range, $0<b<1$. Further inspection of $\varepsilon_{1}, J_{i+1}$ and $K_{i+1}$ in (1c) and (1d), shows that the only remaining poles are due to the zeros of $\left(1+p_{i} \cot \left(\Phi_{i}\right)\right)$ and $\left(1+p_{i+1} \cot \left(\Phi_{i+1}\right)\right)$, which appear in the denominators of these parameters. It can be shown that neither of $1 /\left(1+p_{i} \cot \left(\Phi_{i}\right)\right)$ nor $1 /\left(1+p_{i+1} \cot \left(\Phi_{i+1}\right)\right)$ have zeros in the range $0<b<1$, under the above constraints on $V_{i}, \eta_{i}, a_{i}$, and $b$. Thus, these quantities result in redundant poles which may safely be removed without changing the zeros of the original dispersion function, $\varepsilon_{M}$. The result of removing these poles is that the dispersion equation reduces to,

$$
\chi_{M}=0 \text {, }
$$

where the modified dispersion function, $\chi_{M}$, satisfies the recurrence relation,

with,

$$
\chi_{i+1}=D_{i+1} \chi_{i}-E_{i+1} \chi_{i-1} \text {, }
$$

$$
\begin{aligned}
& D_{i+1}=\left(\cot \left(\Phi_{i+1}\right)-p_{i+1}\right)+\mu_{i, i+1}\left(\cot \left(\Phi_{i}\right)+p_{i}\right) e^{-2 V_{i, i+1} \sqrt{b}} \\
& E_{i+1}=\mu_{i, i+1} \csc ^{2}\left(\Phi_{i}\right) e^{-2 V_{i, i+1} \sqrt{b}} .
\end{aligned}
$$

These recurrence parameters take more simple forms compared to $J_{i+1}$ and $\mathrm{K}_{i+1}$, in (1c) and (1d). The dispersion functions, $\chi_{0}=1$ and $\chi_{1}=\left(\cot \left(\Phi_{1}\right)-p_{1}\right)$. Unlike $\varepsilon_{M}$, the modified dispersion function, $\chi_{M}$, has no singularities in the range, $0<b<1$, in the case of single-mode waveguide arrays. Thus, the change of the sign of $\chi_{M}$ around any point $b$ in that range only implies a zero at that point. This continuity of $\chi_{M}$ simplifies searching for the roots of the dispersion equation. 


\section{DIVERSITY OF DISPERSION FUNCTIONS}

Thus far, the dispersion function has been modified by eliminating redundant poles from the original dispersion function. Further modification of the dispersion function may be carried out by first writing the dispersion function (2b) in terms of the determinant of a tridiagonal matrix. Next by noting that $E_{i+1}$ in (2d) does not have any zeros in the entire $0<b<1$ range, which allows dividing each raw of this determinant by the off-diagonal elements, without changing the zeros of the dispersion function. This modification allows generating different dispersion functions which are all continuous and share the same zeros in the range, $0<b<1$. For example, one form of the dispersion equation is given by,

$$
\delta_{M}=0,
$$

where the dispersion function, $\delta_{M}$, satisfies the recurrence relation,

$$
\delta_{i+1}=A_{i+1} \delta_{i}-B_{i+1} \delta_{i-1} \text {, }
$$

with the following recurrence parameters,

$$
\begin{aligned}
A_{i+1}= & \left\{\left(\cot \left(\Phi_{i+1}\right)-p_{i+1}\right) / \sqrt{\mu_{i, i+1}}\right) \sin \left(\Phi_{i}\right) e^{V_{i, i+1} \sqrt{b}} \\
& +\sqrt{\mu_{i, i+1}}\left(\cos \left(\Phi_{i}\right)+p_{i} \sin \left(\Phi_{i}\right)\right) e^{-V_{i, i+1} \sqrt{b}} \\
B_{i+1}= & \left(\sqrt{\mu_{i, i+1} / \mu_{i-1, i}}\right)\left(\sin \left(\Phi_{i-1}\right) / \sin \left(\Phi_{i}\right)\right) e^{-\left(V_{i, i+1}-V_{i-1, i}\right) \sqrt{b}},
\end{aligned}
$$

and the dispersion functions, $\delta_{1}=\left\{\left(\cos \left(\Phi_{1}\right)-p_{1} \sin \left(\Phi_{1}\right)\right) / \sqrt{\mu_{1,2}}\right\} e^{V_{1,2} \sqrt{b}}$ and $\delta_{0}=1$. Indeed, $\delta_{1}, \chi_{1}$, and $\varepsilon_{1}$ all have the same zeros in the range, $0<b<1$. The particular form of the dispersion function defined by (3b)-(3d) has an advantage over other forms for uniform arrays where $B_{i+1}=1$ and only one recurrence parameter, i.e. $A_{i+1}$, is required to compute the dispersion function.

\section{NUMERICAL EXAMPLES}

The continuity of $\delta_{M}$ in the range, $0<b<1$, eliminates the problem of bracketing singularities in that range and allows the zeros of the dispersion function at one recurrence step to bracket its zeros at the next recurrence step. One of the primary goals of this paper is to use this RZB technique to find the roots of the dispersion equation. The following numerical examples apply this technique to compute the effective indexes of the TE and TM guided modes of both uniform and non-uniform waveguide arrays.

The first example uses a non-uniform array of $M=4$ single-mode waveguides with the following design parameters. The substrate refractive index $n_{s}=1.5$. The core refractive indexes of the isolated waveguides are $n_{1}=1.55, n_{2}=1.54, n_{3}=1.56$, and $n_{4}=1.53$. The core widths are, $d_{1}=1.3 \mu \mathrm{m}, d_{2}=1.1 \mu \mathrm{m}, d_{3}=1.0 \mu \mathrm{m}$, and $d_{4}=1.5$, while the separation between the waveguides are, $d_{1,2}=2 \mu \mathrm{m}, d_{2,3}=3 \mu \mathrm{m}$, and $d_{3,4}=1 \mu \mathrm{m}$. Table I shows the result of computations of the effective indexes of the TE and TM 
modes of the array at a free-space wavelength, $\lambda_{o}=1.3 \mu \mathrm{m}$. In these computations the RZB technique was applied with the bisection method [7] to compute the zeros of both, $\delta_{M}$ and $\varepsilon_{M}$, given by (3b) and (1b), respectively. The results of computations are compared with those obtained by a beam propagation method (BPM) simulator which employs an iterative technique with transparent boundary conditions [10]. The BPM computations used a computational window of $40 \mu \mathrm{m}$, a grid size, $\Delta x=0.001 \mu \mathrm{m}$, a step size in the propagation direction, $\Delta z=0.5 \mu \mathrm{m}$, and an overall propagation length of 5 $\mathrm{mm}$. It is shown that the RZB technique is successful in computing the zeros of $\delta_{M}$, and consequently the roots of the dispersion equation (3a), for both TE and TM modes. It fails to compute the roots of (1a) because of the presence of singularities in the dispersion function, $\varepsilon_{M}$.

The second example uses a uniform array of $M=8$ single-mode waveguides each of core refractive index 1.55, substrate refractive index 1.5, core width $1 \mu \mathrm{m}$, and waveguide separation $3 \mu \mathrm{m}$. As before, the effective indexes of TE and TM modes were computed using the RZB at a free-space wavelength, $\lambda_{o}=1.3 \mu \mathrm{m}$, with both the modified and conventional dispersion functions, $\delta_{M}$ and $\varepsilon_{M}$. In computing the zeros of $\delta_{M}$ only one recurrence parameter was used; see section III. The results of Table II show excellent agreement between the modal indexes computed by the RZB technique using the modified dispersion function, $\delta_{M}$, and those obtained by the iterative BPM technique. However, the RZB technique fails in computing the zeros of $\varepsilon_{M}$ due to the discontinuity of this function. The BPM computations used a computational window of $70 \mu \mathrm{m}$, $\Delta x=0.01 \mu \mathrm{m}, \Delta z=1 \mu \mathrm{m}$, and the same propagation length of the previous example. The small difference between the modal indexes of the successive modes verify the utility of the RZB technique in finding closely spaced roots of the dispersion equation.

\section{References}

[1] H. Nishihara, M. Haruna, T. Sukara, Optical Integrated Circuits, McGraw-Hill, 1989.

[2] Y.-F. Li and J.W.Y. Lit, "Generalized dispersion properties of a multilayer dielectric planar waveguide," J. Opt. Soc. Am. A, vol.9, pp.121-129, 1992.

[3] L. M. Walpita, "Solutions for planar optical waveguide equations by selecting zero elements in a characteristic matrix," J. Opt. Soc. Am. A, vol.2, pp.595-602, 1985.

[4] K.S. Chiang, "Coupled-zigzag-wave theory for guided waves in slab waveguide arrays," J. Lightwave Technol., vol.10, pp.1380-1387, 1992.

[5] E. Anemogiannis, and E.N. Glytsis, "Multilayer waveguides: Efficient numerical analysis of general structures," J. Lightwave Technol., vol.10, pp.1344-1351, 1992. 
[6] C. Chen, P. Berini, D. Feng, S. Tanev, and V.P. Tozolov, "Efficient and accurate numerical analysis of multilayer planar optical waveguides," Opt. Express, vol. 7, pp. 260-272, 2000.

[7] W.H. Press, S.A. Teukolsky, W.T. Vetterling, and B.P. Flannery, Numerical Recipes in C: The Art of Scientific Computing, $2^{\text {nd }}$ ed. New York: Cambridge University Press, 1996.

[8] C. Ma, "Coupling properties in periodic waveguides and in multiple quantum-well waveguides," IEEE J. Quantum. Electron., vol.30, pp. 2811-2816, 1994.

[9] The MathWorks, Inc., Natick, MA, U.S.A.

[10] G.R. Hadley and R.E. Smith, "Full-vector waveguide modeling using an iterative finite difference method with transparent boundary conditions", IEEE J. Quantum. Electron., vol.13, pp. 465-469, 1995.

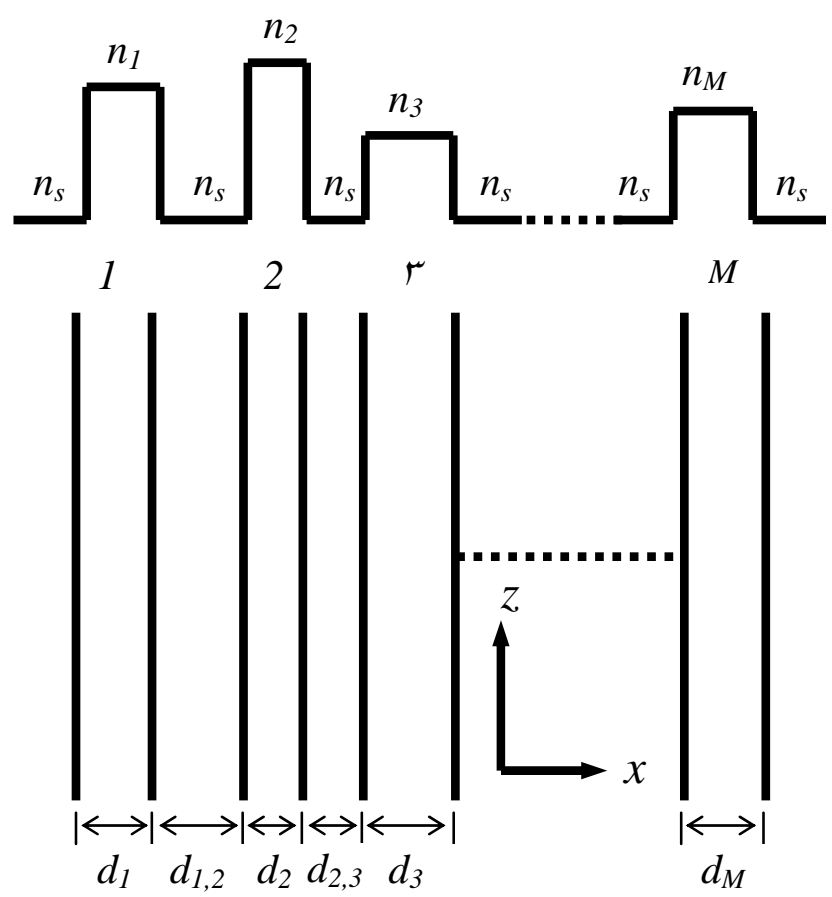

Fig.1. Schematic of an array of $M$-coupled waveguides (bottom) with the corresponding refractive index profile (top). 
TABLE I

COMPUTED VALUES OF MODAL EFFECTIVE INDEXES OF TE AND TM MODES AT $\lambda_{0}=1.3 \mu \mathrm{m}$ FOR A NONUNIFORM ARRAY OF $M=4$ SINGLE-MODE WAVEGUIDES

\begin{tabular}{|c|c|c|c|}
\hline \multirow{2}{*}{ mode } & \multicolumn{3}{|c|}{$N$} \\
\hline & RZB + Eq.(3a) & BPM & RZB + Eq.(1a) \\
\hline $\mathrm{TE}_{0}$ & 1.529001 & 1.529000 & 1.528142 \\
\hline $\mathrm{TE}_{1}$ & 1.527431 & 1.527431 & 1.521509 \\
\hline $\mathrm{TE}_{2}$ & 1.516728 & 1.516727 & 1.511549 \\
\hline $\mathrm{TE}_{3}$ & 1.513257 & 1.513259 & 1.500719 \\
\hline $\mathrm{TM}_{0}$ & 1,047733 & 1.527734 & 1.528142 \\
\hline $\mathrm{TM}_{1}$ & $1,0 Y 701 \mathrm{r}$ & 1.526587 & 1.521174 \\
\hline $\mathrm{TM}_{2}$ & $1,017.77$ & 1.516068 & 1.512809 \\
\hline $\mathrm{TM}_{3}$ & $1,01 Y \wedge . \varepsilon$ & 1.512808 & 1.502936 \\
\hline
\end{tabular}

TABLE II

COMPUTED VALUES OF MODAL EFFECTIVE INDEXES OF TE AND TM MODES AT $\lambda_{0}=1.3 \mu \mathrm{m}$ FOR A UNIFORM ARRAY OF $M=8$ SINGLE-MODE WAVEGUIDES

\begin{tabular}{|c|c|c|c|}
\hline \multirow{2}{*}{ mode } & \multicolumn{3}{|c|}{$N$} \\
\hline & RZB + Eq. (3a) & BPM & RZB + Eq.(1a) \\
\hline $\mathrm{TE}_{0}$ & 1,OYAVVE & 1.528751 & $1,0 \leq 977 \leqslant$ \\
\hline $\mathrm{TE}_{1}$ & 1,OYAOT & $1.5280 \leqslant 1$ & $1,0 \leq 7701$ \\
\hline $\mathrm{TE}_{2}$ & $1,0 Y 1101$ & $1.52810 \leq$ & $1,0 Y \wedge V \vee A$ \\
\hline $\mathrm{TE}_{3}$ & $1,0 Y \times 701$ & $1.52 \times 709$ & 1,OrOV.r \\
\hline $\mathrm{TE}_{4}$ & $1,0 Y V 1 \ldots$ & $1.52 \vee \cdot 99$ & $1,0 . \leqslant T r V$ \\
\hline $\mathrm{TE}_{5}$ & 1,0YTorV & ס סדים 1.527 & $1,0.11 \leq r$ \\
\hline $\mathrm{TE}_{6}$ & $1,0 Y T . \leqslant V$ & $1.527 \cdot \leq 1$ & $1,0 \ldots \leqslant \leqslant 1$ \\
\hline $\mathrm{TE}_{7}$ & 1,0Y०V1. & $1.520 \mathrm{VYO}$ & $1,0 \ldots$. \\
\hline $\mathrm{TM}_{0}$ & 1,0rV99. & 1.527974 & $1,0 \leqslant 91 \cdot r$ \\
\hline $\mathrm{TM}_{1}$ & 1,OYVVTA & 1.527751 & $1,0 \leq \wedge 717$ \\
\hline $\mathrm{TM}_{2}$ & 1,OYVTMV & 1.527345 & $1,0 \leq Y M T$. \\
\hline $\mathrm{TM}_{3}$ & 1,047119 & 1.52 & 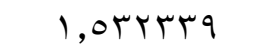 \\
\hline $\mathrm{TM}_{4}$ & 1,0YTrYq & 1.527rTs & $1,0 Y \cdot \lambda \leq 1$ \\
\hline $\mathrm{TM}_{5}$ & 1,0Y07T & דוrד 1.520 & $1,0 \cdot \vee \wedge \leq 7$ \\
\hline $\mathrm{TM}_{6}$ & 1,040111 & 1.520111 & $1,0 \ldots \wedge V r$ \\
\hline $\mathrm{TM}_{7}$ & $1,0 Y \leqslant V \leqslant 9$ & $1.52 \leqslant \vee 79$ & $1,0 \ldots \leq 7$ \\
\hline
\end{tabular}

